\title{
Genotype-guided dosing of coumarin derivatives: the European pharmacogenetics of anticoagulant therapy (EU-PACT) trial design
}

The narrow therapeutic range and wide interpatient variability in dose requirement make anticoagulation response to coumarin derivatives unpredictable. As a result, patients require frequent monitoring to avert adverse effects and maintain therapeutic efficacy. Polymorphisms in VKORC1 and CYP2C9 jointly account for about $40 \%$ of the interindividual variability in dose requirements. To date, several pharmacogeneticguided dosing algorithms for coumarin derivatives, predominately for warfarin, have been developed. However, the potential benefit of these dosing algorithms in terms of their safety and clinical utility has not been adequately investigated in randomized settings. The European Pharmacogenetics of Anticoagulant Therapy (EU-PACT) trial will assess, in a single-blinded and randomized controlled trial with a follow-up period of 3 months, the safety and clinical utility of genotype-guided dosing in daily practice for the three main coumarin derivatives used in Europe. The primary outcome measure is the percentage time in the therapeutic range for international normalized ratio. This report describes the design and protocol for the trial.

\section{KEYWORDS: acenocoumarol CYP2C9 dosing algorithm EU-PACT pharmacogenetics phenprocoumon randomized controlled trial VKORC1 warfarin}

Coumarin derivatives, such as warfarin, acenocoumarol and phenprocoumon, are commonly prescribed as oral anticoagulants for the treatment of thromboembolic disorders. Therapy with coumarin derivatives is most effective when the international normalized ratio (INR) is kept within a narrow range. Prescribing of these drugs is difficult because of their narrow therapeutic window and the wide interindividual variability in dose requirement; coumarin derivative dosages can vary by a factor 10 among patients [1,2]. For these reasons, it is of difficult to predict anticoagulation response to a standard dosing regimen, as this is the case with the current dosing algorithms used for the initiation of anticoagulation therapy. Consequently, treatment is often either subtherapeutic (due to underdosing) or supratherapeutic (due to overdosing), placing the patient at risk of (recurrent) thrombosis or hemorrhage, respectively, which can be life-threatening [3,4]. Because of the uncertainty in anticoagulation response, patients on coumarin derivative therapy require careful monitoring $[1,4,5]$.

Anticoagulation response to coumarin derivatives is influenced by a number of clinical, environmental and genetic factors. It has been established that factors such as concurrent drug therapy, co-morbidity, age, sex, BMI, smoking and dietary vitamin $\mathrm{K}$ intake influence coumarin derivative dose requirements [6-10]. Polymorphisms in the CYP2C9 and VKORC1 genes, encoding for the metabolizing enzyme cytochrome P450 2C9 (CYP2C9) and the target enzyme vitamin $\mathrm{K}$ epoxide reductase (VKOR) respectively, together account for about $40 \%$ of the variability in coumarin derivative maintenance dose requirements $[11,12]$. Several studies have demonstrated that patients with allelic variants in the CYP2C9 and VKORC1 genes require lower coumarin derivatives doses than those with wild-type alleles [13-17]. This exposes them to a greater risk of over-anticoagulation and hemorrhage, particularly during initiation of therapy. Polymorphisms in other genes, for example, the CYP4F2 gene, have only a nominal effect on the coumarin derivate dose $[18,19]$.

The recognition that genetic factors influence coumarin derivative dose requirements and thus may predispose to serious and life threatening hemorrhage has highlighted the inadequacy of the currently used dosing regimens. To date, several studies have quantified the contribution of the CYP2C9 and VKORC1 genes in coumarin derivative dose requirement and put forward pharmacogenetic-based dosing equations [10,12,20-25]. However, these equations (mainly developed for warfarin) are based on data derived from patients on stable maintenance therapy and are therefore unsuitable for those
Rianne MF van Schie,

Mia Wadelius,

Farhad Kamali,

Ann K Daly,

Vangelis G

Manolopoulos,

Anthonius de Boer,

Rita Barallon,

Talitha I Verhoef,

Julia Kirchheiner,

Elisabeth Haschke-

Becher, Montserrat Briz,

Frits R Rosendaal,

William K Redekop ${ }^{1}$,

Munir Pirmohamed \&

Anke-Hilse Maitland-van

der Zee

${ }^{+}$Author for correspondence:

Utrecht University, Faculty of

Science, PO Box 80082

3508 TB Utrecht,

The Netherlands

Tel.: +31 302534042

Fax: +31302539166

a.h.maitland@uu.nl

For a full list of affiliations,

please see back page

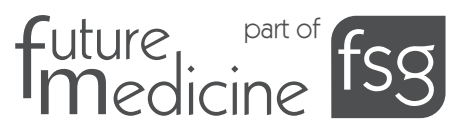


commencing oral anticoagulation therapy, where loading doses often are used. The challenge in demonstrating the benefits of pharmacogeneticguided dosing lies in the development of dosing algorithms, which can improve the accuracy of dosing during both the initiation of therapy and subsequent maintenance therapy. A pharmacogenetic approach to oral anticoagulation therapy requires two things: robust dosing algorithms that allow for prediction of loading and maintenance doses developed from verification data and validated in a replication set; and also that the clinical validity and utility of the dosing algorithms is tested within a randomized controlled trial (RCT) setting. The cost-effectiveness of genotype-guided dosing must also be examined, since current available cost-effectiveness analyses (CEA) do not point in the same direction [26-28]. Additional clinical trials are necessary to prove the clinical relevance and cost-effectiveness of pretreatment genotyping before implementing this approach in clinical practice.

The European Pharmacogenetics of Anticoagulant Therapy (EU-PACT) trial will assess the safety, clinical utility and cost-effectiveness of the newly developed pharmacogenetic-guided dosing algorithms for acenocoumarol, phenprocoumon and warfarin. The trial will take place in seven countries in Europe and is supported by the European Commission FP7 Programme.

\section{Materials \& methods - Design \& setting}

The EU-PACT study is a two-armed, singleblinded (patients are blinded), RCT which will test the effectiveness of dosing regimens that include genetic factors compared with dosing regimens without these factors. This will be evaluated during the first 3 months after initiation of anticoagulation therapy in patients treated with the three different coumarin derivatives warfarin, acenocoumarol, or phenprocoumon. The study will be performed in 13 different centers, covering general practitioners, anticoagulation clinics and hospitals. These centers are located in seven European countries, namely the UK, Sweden, the Netherlands, Spain, Greece, Germany and Austria. It will take 2 years to complete the inclusion of patients.

\section{Sample size calculation}

Based on data from the first 3 months of warfarin therapy in Sweden and the UK, the mean percentage time in range (\%TIR) of the INR ranges from 52 to $65 \%$ in large cohorts of patients with atrial fibrillation (AF) or venous thrombosis
(VT). In other participating centers, similar values for acenocoumarol and phenprocoumon are found. For example, in the Netherlands, where predominately acenocoumarol and phenprocoumon are used, the average range of \%TIR of the INR across different anticoagulation clinics varies from 22.5 to $56.5 \%$ (median $38.0 \%$ ) for the first 8 weeks of coumarin derivative therapy and from 32.5 to $67.0 \%$ (median $53.5 \%$ ) in patients anticoagulated short-term (2-6 months therapy) [101]. With $80 \%$ power and at $5 \%$ significance level, a total of 442 patients each in the intervention and control groups will be needed in order to demonstrate a $5 \%$ greater $\%$ TIR of the INR. Assuming a $10 \%$ dropout after study entry, 985 patients will need to be recruited for each coumarin derivative, making a total of 2955 patients randomized for the three trials.

\section{Study population}

Newly diagnosed patients with either AF or VT, that is, pulmonary embolism (PE) or deep venous thrombosis (DVT), requiring anticoagulant therapy with acenocoumarol, phenprocoumon or warfarin are eligible for the trial if they meet the inclusion and exclusion criteria.

\section{Inclusion \& exclusion criteria}

Patients of both sexes, aged 18 years or older, diagnosed with AF or VT requiring coumarin derivative therapy with a target INR in the lower intensity range $(2.0-3.5$ in the Netherlands and 2.0-3.0 in the other participating countries) for at least 12 weeks are eligible for the trial. They must have the ability to attend the scheduled visits and have to provide written informed consent.

Patients will be excluded from the trial if they have been treated with a coumarin derivative previously, if their CYP2C9 or VKORC1 genotype is known, if they are pregnant or breastfeeding, or if they suffer from severe cognitive impairment. The presence of a mechanical heart valve will also lead to exclusion. Another exclusion criterion is an abnormal clotting function at baseline INR, that is a baseline INR of 1.5 and higher, a platelet count less than $100 \times 10^{9} \mathrm{~L}^{-1}$ or an activated partial thromboplastin time (APTT) more than 1.3-times upper reference value that is not explained by the presence of lupus anticoagulants.

\section{Patient allocation \& treatment}

Patients will be randomized to either the intervention group, which will be dosed according to a genotype-guided dosing algorithm, or to the control group, which will be dosed according to 
a dosing regimen without genotype. All dosing regimens will be computer assisted. The genotype-guided dosing algorithm will include the patient's genetic information, clinical and demographic data and in the monitoring phase the previous INR. The acenocoumarol and phenprocoumon control groups will be dosed according to a nongenotype-guided dosing algorithm, which uses the same parameters of the dosing algorithm in the intervention group except for the CYP2C9 and VKORC1 genotypes. The warfarin control group will be dosed according to standardized clinical care. A schematic presentation of the study design is given in Figure 1 .

The dosing algorithms for all three coumarin derivatives will be developed with various datasets $[29,30]$ which contain information about factors known to influence dose requirements such as age, sex, CYP2C9 and VKORC1 genotype, height and weight. All dosing algorithms are divided into two subalgorithms; the loading dose algorithm and the monitoring dose algorithm. The monitoring dose algorithms for acenocoumarol and phenprocoumon will be developed with linear regression. The warfarin monitoring dose algorithm is based on the model developed by the International Warfarin Pharmacogenetics Consortium [21]. Loading doses for all three coumarin derivatives will be calculated from the monitoring dose using pharmacokinetic information of the coumarin derivative for each genotype.

\section{Blinding \& randomization}

The EU-PACT trial is a single-blinded study with patients being blinded to the study treatment. Staff with access rights to the database will be able to view all data on patients recruited in their own center, except for genotype in the control patients.

Eligible patients who consent to take part in the study will undergo medical screening prior to enrollment. Patient demographics and clinical data including age, gender, height, weight and information on co-morbidity (e.g., malignancies and thyroid disease), co-medication and alcohol intake will be recorded. Following a successful screen and fulfillment of the study inclusion

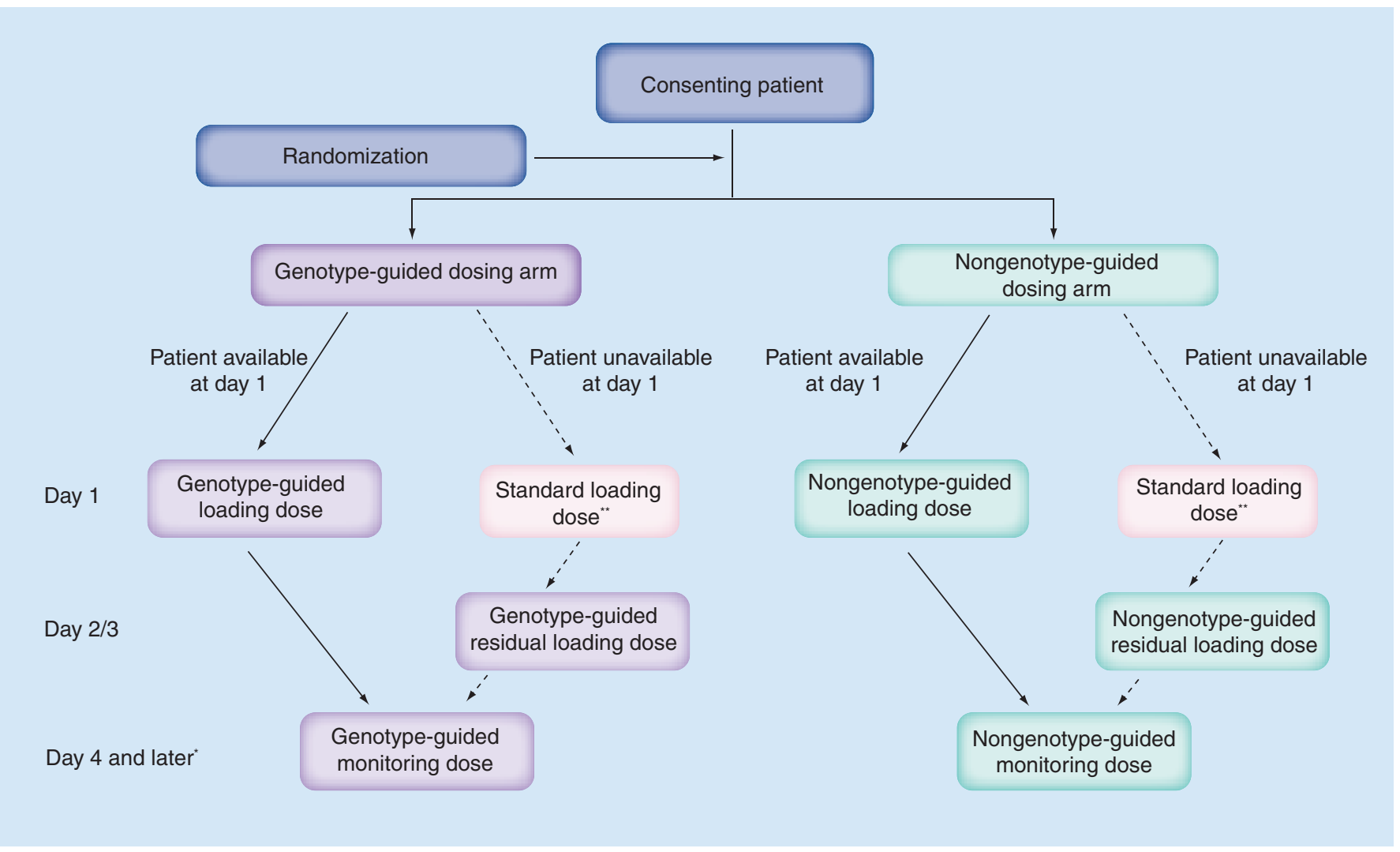

Figure 1. Schematic presentation of the study design as for each coumarin derivative.

*The loading dose algorithm is optimally followed for 3 days, and the start of the monitoring dose algorithm on day 4 . However, due to weekends the monitoring dose algorithm may also start on day 3, 5 or 6.

${ }^{* *}$ In exceptional cases (shown by the dashed arrow), if the patient is randomized to either the intervention or the control group later than day 1 , and the patient has received coumarin dosing according to usual clinical care only at day 1 , the patient may receive a loading dose according to one of the loading dose algorithms for the two days remaining until the next planned international normalized ratio test. 
criteria, patients will be randomized to the intervention or control group by block randomization per study center.

\section{Patient consent \& information}

All patients will be informed verbally and in writing about the aims of the study and how participation would affect their treatment. Standard information about the impact of diet (such as vitamin $\mathrm{K}$ intake and alcohol) and interacting drugs (e.g., CYP2C9 inducers and inhibitors) and the possible hazards associated with the therapy will be provided to all patients.

\section{Genotyping}

A blood sample will be taken from all participating patients for genotyping of $C Y P 2 C^{*} 2$ (rs1799853), CYP2C9*3 (rs1057910) and VKORC1-1639G>A (rs9923231) prior to the commencement of oral anticoagulation therapy. Genotyping will be performed using a new rapid method which provides results within $1.5 \mathrm{~h}$. The method employs a HyBeacon ${ }^{\circledR}$ technology (LGC Ltd, Middlesex, UK) [31-33], which will be used in combination with Optigene's Genie 1 instrument (Optigene Ltd, Horsham, UK) as a point-of-care test in a nonlaboratory environment. An aliquot of blood will be stored for quality control analysis of the point-of-care test.

The blood samples from patients who are found to be either sensitive or resistant to a coumarin derivative will later be subjected to further genetic analysis through sequencing approaches for the identification of rare mutations in genes mediating the pharmacology or disposition of coumarin derivatives and other novel genes to identify the genetic basis of discordant phenotypes [34] .

\section{Treatment procedures}

Patients suffering from VT who require acute anticoagulation will initially be treated with (parenteral) low molecular weight heparin in combination with the coumarin derivative. Low molecular weight heparin will be discontinued according to local guidance. Patients with AF initiating anticoagulation for stroke prophylaxis will be administered coumarin derivatives alone.

The loading dose will be calculated according to the loading dose algorithm for the prescribed coumarin derivative. If a patient for some reason does not receive the individualized starting dose (e.g., because of weekends), (s)he can obtain an adjusted loading dose regimen on day 2 . After the first INR determination (preferably on day 4), subsequent dosing will be calculated according to the monitoring dose algorithm. The monitoring dose algorithm is based on the same factors as the loading dose algorithm including the patient's previous INR.

The use of all concurrent medications will be recorded, including the drug's trade name, dosage, start and end date and indication for treatment.

\section{Alcohol intake, quality of life \& compliance assessment}

Alcohol intake (alcohol use disorders identification test [AUDIT]) [35] and quality of life (EQ-5D) [36] will be assessed at the patient's first and last study visits. To monitor patient compliance to treatment, patients will complete a medication questionnaire at each visit.

\section{- Assessment of safety}

International normalized ratio measurements will be carried out on days 1, 4, 6, 8, 15, 22, 57 and 85 (days 4-85 may be adjusted slightly) to ensure that patients are adequately anticoagulated. If clinically needed, additional INR measurements will be performed.

Any undesired medical event, not necessarily related to the use of coumarin derivatives, is defined as a serious adverse event if it leads to death; is life-threatening, for example, a major hemorrhage; requires (prolonged) hospitalization or is a congenital anomaly/birth defect [102] Hemorrhages will be categorized into major and minor according to the International Society on Thrombosis and Haemostasis (ISTH) classification of hemorrhagic events [37]. The algorithm of Naranjo et al. will be used to determine the probability that any observed (serious) adverse event is associated with the trial treatment [38]. Safety data will be evaluated by an independent Data and Safety Monitoring Board (DSMB).

\section{Patient withdrawal}

At any time, patients are free to withdraw from the trial without giving a reason. The patient will be contacted to obtain information about the reason(s) for withdrawal and any experienced adverse events (AEs). The date and reason for the withdrawal will be reported in the case report form (CRF). Each patient withdrawn less than 2 weeks after study entry will be replaced by a new one.

The investigator will be able to withdraw patients from the trial for safety reasons, for example, due to AEs that contraindicate continued participation, pregnancy or a deteriorated general condition.

After participation in the trial, anticoagulation therapy for each patient will be continued according to individual needs and local protocols. 


\section{Ethics}

The EU-PACT study will be performed according to the study protocol, International Conference on Harmonisation Good Clinical Practice (ICHGCP), the Declaration of Helsinki, EU directives and applicable regulatory requirements. The study will be submitted for approval to medical review ethics committees in all participating countries.

\section{Study outcomes}

\section{Primary outcome}

The primary outcome of the study is the \%TIR of the INR, INR range 2.0-3.0, during the first three months following initiation of anticoagulant therapy calculated by the interpolation method [39].

\section{Secondary outcomes}

The secondary outcomes for the first 3 months of therapy include:

- Time to and number of patients with INR 4.0 or above, which indicates over-anticoagulation;

- Percentage of time spent with INR 4.0 or above;

- Percentage of time spent with INR 1.5 or less, which indicates under-anticoagulation;

- Time to reach therapeutic INR defined as the time to the first INR within target range, provided that a subsequent INR measured at least 1 week later is also within target range;

- Time to reach stable dose defined as time to reach an unchanged dose ( $<10 \%$ change) at consecutive visits and the INR being within the target range for a period of at least 3 weeks;

- Time to and number of minor and major hemorrhages;

- Time to and number of thromboembolic events;

- The occurrence of coumarin derivative hypersensitivity defined as dose requirements of $1.5 \mathrm{mg}$ warfarin/day or less, $1.5 \mathrm{mg}$ phenprocoumon/day or less or $1.0 \mathrm{mg}$ acenocoumarol/day or less during maintenance;

- The occurrence of coumarin derivative resistance defined as dose requirements of at least $10 \mathrm{mg}$ warfarin/day, at least $6 \mathrm{mg}$ phenprocoumon/day or at least $8 \mathrm{mg}$ acenocoumarol/day during maintenance;

- Number of coumarin derivative dose adjustments;

- The utility of the LGC Ltd's rapid genotyping test in daily anticoagulation practice;
- Patient quality of life;

- The cost-effectiveness of pharmacogeneticguided dosing for each of the three coumarin derivatives.

\section{Data collection}

Information on each patient's age, gender, height, weight, alcohol intake, co-morbidity (e.g., malignancies and thyroid disease), and co-medication will be obtained either directly from the initial interview with the patient or the self-completed questionnaires and from medical records or pharmacy records whenever possible. Other parameters including INR values, number of INR measurements, coumarin derivative doses, the number of dose changes, changes in concurrent disease or drug therapy, laboratory data and any adverse events experienced during the study will be recorded during the course of the trial.

\section{Data management}

Data collection and storage will be done using Promasys (Promasys, Leiden, The Netherlands), a data management software system which supports multicenter trials. The central functionality of this system is related to data management, which allows the setting up of a database structure into which the clinical trial data can be entered thereby preserving the integrity of the data captured. Each center can log on to the central server and enter data live in the electronic CRF.

\section{Statistical analysis}

The trial results will be evaluated according to the intention-to-treat and the per-protocol analysis. Estimation of mean differences (plus 95\% CIs) in \%TIR of the INR between genotype and nongenotype-guided dosing, when necessary with correction for confounding variables, will be performed with linear regression for each coumarin derivative. For the remaining outcomes, appropriate hypothesis tests will be adopted to test for differences between the two study arms, for example the Cox-proportional hazard model for dichotomous outcomes. These outcomes will also be presented using Kaplan-Meier curves. The nominal p-value for assessing statistical significance will be 0.05 , although this will be adjusted for the number of comparisons made in order to conserve the type I error rate. All hypothesis tests will be two-sided. We will analyze whether the results found are similar for all three anticoagulation drugs and whether they are similar within and 
between the different study centers. For the latter, multilevel regression models stratified by center will be used. To be able to extrapolate our data to the whole EU these comparisons are of the utmost importance.

\section{Cost-effectiveness analysis}

A CEA for the individualized dosing regimen will be performed according to established methods [103]. The primary analysis will utilize the societal perspective, meaning that all costs will be included in the CEA regardless of who incurs these costs. As a consequence, we will include not only health service costs (e.g., from ambulatory care, hospital care and medications), but also costs incurred by patients. Two types of health outcomes will be examined: the incidence of adverse events (hemorrhage, thromboembolic events); and the quality-adjusted life-years (QALYs), measured using the EQ-5D. Two sets of CEA will be performed: a short-term CEA and a longterm CEA. The short-term CEA will focus on the clinical results during the study's 3-month follow-up period. We will perform a long-term CEA, which will be possible by creating a model that combines the results of this RCT with data from other clinical, epidemiological and health services research studies. We plan to analyze coumarin derivative-specific and country-specific data to estimate resource use and unit costs.

\section{Discussion}

Current strategies for initiation of oral anticoagulation therapy are inadequate, exposing patients to a risk of (recurrent) thrombosis owing to underdosing or hemorrhage owing to overdosing. Polymorphisms in CYP2C9 and $V K O R C 1$ genes have a major impact on coumarin derivative dose requirements. It is anticipated that pharmacogenetic-guided dosing will improve the safety of anticoagulation therapy with coumarin derivatives through improved accuracy of dosing. The three small scale prospective studies reported to date have not convincingly demonstrated the potential benefit of pharmacogenetic-guided dosing on treatment outcomes [40-42]. One of these studies included both CYP2C9 and VKORC1 polymorphisms [41] whilst the other two only included polymorphisms in CYP2C9 in the dosing algorithms used [40,42]. Inclusion of the VKORC1 gene in a pharmacogenetic-guided dosing algorithm is likely to improve the accuracy of dosing given that $V K O R C 1$ polymorphisms explain up to a third of the interindividual variability in coumarin derivative dose requirements. Moreover, reported ongoing randomized trials of genotype-guided dosing as well as the aforementioned small prospective studies only evaluate pharmacogenetic-guided dosing with warfarin [43]. In contrast, the EU-PACT trial will be evaluating a pharmacogenetic approach to anticoagulation therapy with the three most prescribed coumarin derivatives in large patient cohorts in seven European countries using pharmacogenetic-based dosing algorithms which include both CYP2C9 and VKORC1 genes. The EU-PACT trial results should therefore be widely applicable to patients across Europe and elsewhere. Unlike previous prospective studies, the EU-PACT trial will make use of rapid point-of-care genotyping, thus making it possible to commence genotype-guided therapy straight away.

It is anticipated that initiation of anticoagulation therapy using a genotype-guided dosing regimen allows the patient to reach target INR quickly and to remain within the therapeutic range more effectively. Although hemorrhages are clinically more relevant than percentage time within target INR, we chose \%TIR rather than hemorrhages as the primary study outcome. It would not be financially and logistically possible to collect the considerably larger number of patients needed in order to detect a difference in hemorrhages. It is well established that maintaining anticoagulation within the therapeutic range is critical for therapeutic efficacy and safety. Several studies have shown that there is a close relationship between the INR and the risk of hemorrhage and thrombotic events; the risk of hemorrhage increases markedly for supratherapeutic INR values [44-46], and the risk of death owing to cerebral hemorrhage doubles for every unit increase in INR [47], while the risk of a thrombotic event increases with subtherapeutic INR values $[4,45]$. Because of this close association between INR and these outcomes, it is expected that our chosen primary outcome is a good indicator of treatment safety outcome.

\section{Conclusion}

The goal of this study is to evaluate the added value and the cost-effectiveness of pharmacogenetic-guided dosing of coumarin derivatives in daily practice. To our knowledge, EU-PACT is the first large scale randomized controlled trial of pharmacogenetic-guided anticoagulation therapy ever performed in Europe. 


\section{Future perspective}

Randomization of the first patient is expected in February 2010. Based on the anticipated number of patients available for selection, we expect to obtain full recruitment and follow-up of the last patient by February 2012. Since it is known that $C Y P 2 C 9$ and $V K O R C 1$ polymorphisms are the principal contributors to the interindividual variability in coumarin derivative dose requirements, our hypothesis is that dosing according to a genotype-guided algorithm will increase the safety and efficacy of oral anticoagulation therapy. Cost-effectiveness analyses will be performed to ascertain whether it is economically advantageous to genotype patients before initiating coumarin derivative treatment. Currently, only widely varying estimates of the cost-effectiveness of pharmacogenetics in anticoagulant therapy exist, mostly based on hypothetical assumptions [26-28].

Although the FDA has changed the label of warfarin in the USA [104], this has not led to a change in guidelines by specialist societies such as the American College of Chest Physicians [48], because of the lack of randomized data. Our study, together with the NIHfunded Clarification of Optimal Anticoagulant through Genetics (COAG) trial in the USA [105], aims to fill this evidence gap. Only such positive randomized data is likely to persuade clinicians to incorporate pharmacogeneticbased dosing into their clinical practice. It is also important to state that this is, to the best of our knowledge, the first pharmacogenetic study to assess the utility of a point-of-care diagnostic, an important advance, which, if proven to be effective, would allow pharmacogenetic dosing without a delay, the use and testing of point-ofcare tests in other conditions and drug treatments; and the use of pharmacogenetic dosing beyond the hospital environment, for example, in primary care.

\section{Acknowledgements}

We would like to thank Felix JM van der Meer, Andrea L Jorgensen and Niclas Eriksson for their valuable input. This project is funded by the European Community's Seventh Framework Programme under grant agreement $n^{\circ}$ HEALTH-F2-2009-223062.

\section{Financial \& competing interests disclosure}

The authors have no relevant affiliations or financial involvement with any organization or entity with a financial interest in or financial conflict with the subject matter or materials discussed in the manuscript. This includes employment, consultancies, honoraria, stock ownership or options, expert testimony, grants or patents received or pending, or royalties.

No writing assistance was utilized in the production of this manuscript

\section{Executive summary}

- Insufficient anticoagulation gives rise to an increased risk of (recurrent) thrombosis, while over anticoagulation may lead to hemorrhage.

- Polymorphisms in VKORC1 and CYP2C9 explain about 40\% of the interindividual variability in coumarin derivative dose requirements.

- Many pharmacogenetic-based dosing algorithms have been developed, mainly for warfarin, based on data derived from patients on stable maintenance therapy.

- It is necessary to perform prospective studies to evaluate the added value of a genotype-guided dosing of coumarin derivatives.

- The added value of genotyping prior to coumarin derivative therapy will be investigated for acenocoumarol, phenprocoumon and warfarin in the European Pharmacogenetics of Anticoagulant Therapy (EU-PACT) trial.

- The EU-PACT trial consists of three two-armed, single-blind, randomized controlled trials in which genotype-guided dosing is compared with nongenotype-guided dosing with a follow-up duration of 3 months for each patient.

- To our knowledge, the EU-PACT trial is the first large scale randomized controlled trial of pharmacogenetic-guided anticoagulation therapy ever performed in Europe.

\section{Bibliography}

Papers of special note have been highlighted as:

" of interest

"I" of considerable interest

1 Rosendaal FR: The scylla and charybdis of oral anticoagulant treatment. N. Engl. J. Med. 335, 587-589 (1996).

2 James AH, Britt RP, Raskino CL, Thompson SG: Factors affecting the maintenance dose of warfarin. J. Clin. Pathol. 45, 704-706 (1992).
3 Penning-van Beest FJA, van Meegen E, Rosendaal FR, Stricker BHC:

Characteristics of anticoagulant therapy and comorbidity related to overanticoagulation. Thromb. Haemost. 86, 569-574 (2001).

4 Hylek EM, Skates SJ, Sheehan MA, Singer DE: An analysis of the lowest effective intensity of prophylactic anticoagulation for patients with nonrheumatic atrial fibrillation. $N$. Engl. J. Med. 335, 540-546 (1996).
5 Pirmohamed M: Warfarin: almost 60 years old and still causing problems. Br. J. Clin. Pharmacol. 62, 509-511 (2006).

6 Penning-van Beest FJA, Geleijnse JM, van Meegen E, Vermeer C, Rosendaal FR, Stricker BHC: Lifestyle and diet as risk factors for overanticoagulation. J. Clin. Epidemiol. 55, 411-417 (2002).

7 Carlquist JF, Horne BD, Muhlestein JB et al: Genotypes of the cytochrome $\mathrm{p} 450$ isoform, CYP2C9, and the vitamin K epoxide reductase complex subunit 1 conjointly 
determine stable warfarin dose: a prospective study. J. Thromb. Thrombolysis 22, 191-197 (2006).

8 Schalekamp T, van Geest-Daalderop JHH, Kramer MHH, van Holten-

Verzantvoort ATM, de Boer A: Coumarin anticoagulants and co-trimoxazole: avoid the combination rather than manage the interaction. Eur. J. Clin. Pharmacol. 63, 335-343 (2007).

9 Schalekamp T, Klungel OH, Souverein PC, de Boer A: Increased bleeding risk with concurrent use of selective serotonin reuptake inhibitors and coumarins. Arch. Intern. Med. $168,180-185$ (2008).

10 Gage BF, Eby C, Milligan PE, Banet GA, Duncan JR, McLeod HL: Use of pharmacogenetics and clinical factors to predict the maintenance dose of warfarin. Thromb. Haemost. 91, 87-94 (2004).

- Describes the development of a genotypeguided dosing algorithm. Prospective trials are required to prove effectiveness of the algorithm.

11 Bodin L, Verstuyft C, Tregouet DA et al.: Cytochrome P450 2C9 (CYP2C9) and vitamin $\mathrm{K}$ epoxide reductase (VKORC1) genotypes as determinants of acenocoumarol sensitivity. Blood 106, 135-140 (2005).

" - Showed the correlation between acenocoumarol dosage requirements and polymorphisms in $C Y P 2 C 9$ and VKORC1 first.

12 Wadelius M, Chen LY, Lindh JD et al:: The largest prospective warfarin-treated cohort supports genetic forecasting. Blood 113, 784-792 (2009).

13 Schalekamp T, Brasse BP, Roijers JFM et al.: VKORC1 and CYP2C9 genotypes and acenocoumarol anticoagulation status: interaction between both genotypes affects overanticoagulation. Clin. Pharmacol. Ther. 80, 13-22 (2006).

14 Schalekamp T, Brasse BP, Roijers JFM et al.: VKORC1 and CYP2C9 genotypes and phenprocoumon anticoagulation status: interaction between both genotypes affects dose requirement. Clin. Pharmacol. Ther. 81, 185-193 (2007).

- Showed the correlation between phenprocoumon dosage requirements and polymorphisms in CYP2C9 and VKORC1 first.

15 Reitsma PH, van der Heijden JF, Groot AP, Rosendaal FR, Buller HR: A C1173T dimorphism in the VKORC1 gene determines coumarin sensitivity and bleeding risk. PLoS Med. 2, E312 (2005).
16 Wadelius M, Chen LY, Downes K et al.: Common VKORC1 and GGCX polymorphisms associated with warfarin dose. Pharmacogenomics J. 5, 262-270 (2005).

w- First showed that estimation of the warfarin dosage requirement is influenced by polymorphisms in CYP2C9, VKORC1 and clinical data of the patient.

17 D'Andrea G, D'Ambrosio RL, Di Perna P et al: A polymorphism in the $V K O R C 1$ gene is associated with an interindividual variability in the dose-anticoagulant effect of warfarin. Blood 105, 645-649 (2005).

18 Takeuchi F, McGinnis R, Bourgeois $S$ et al:: A genome-wide association study confirms VKORC1, CYP2C9, and CYP4F2 as principal genetic determinants of warfarin dose. PLoS Genet. 5, E1000433 (2009).

19 Caldwell MD, Awad T, Johnson JA et al.: CYP4F2 genetic variant alters required warfarin dose. Blood 111, 4106-4112 (2008).

20 Schelleman $\mathrm{H}$, Chen J, Chen $\mathrm{Z}$ et al.: Dosing algorithms to predict warfarin maintenance dose in Caucasians and African Americans. Clin. Pharmacol. Ther. 84, 332-339 (2008).

21 Klein TE, Altman RB, Eriksson $\mathrm{N}$ et al.: Estimation of the warfarin dose with clinical and pharmacogenetic data. N. Engl. J. Med. 360, 753-764 (2009).

22 Perini JA, Struchiner CJ, Silva-Assuncao E et al: : Pharmacogenetics of warfarin: development of a dosing algorithm for Brazilian patients. Clin. Pharmacol. Ther. 84 , 722-728 (2008).

23 Sconce EA, Khan TI, Wynne HA et al: : The impact of CYP2C9 and VKORC1 genetic polymorphism and patient characteristics upon warfarin dose requirements: proposal for a new dosing regimen. Blood 106, 2329-2333 (2005).

24 Tham LS, Goh BC, Nafziger A et al: : A warfarin-dosing model in Asians that uses single nucleotide polymorphisms in vitamin $\mathrm{K}$ epoxide reductase complex and cytochrome P450 2C9. Clin. Pharmacol. Ther. 80, 346-355 (2006).

25 Gage BF, Eby C, Johnson JA et al.: Use of pharmacogenetic and clinical factors to predict the therapeutic dose of warfarin. Clin. Pharmacol. Ther. 84, 326-331 (2008).

26 Eckman MH, Rosand J, Greenberg SM, Gage BF: Cost-effectiveness of using pharmacogenetic information in warfarin dosing for patients with nonvalvular atrial fibrillation. Ann. Intern. Med. 150, 73-83 (2009).
27 Schalekamp T, Boink GJJ, Visser LE, Stricker BHC, de Boer A, Klungel OH: CYP2C9 genotyping in acenocoumarol treatment: is it a cost-effective addition to international normalized ratio monitoring? Clin. Pharmacol. Ther. 79, 511-520 (2006).

28 Hughes DA, Pirmohamed M: Warfarin pharmacogenetics: economic considerations. Pharmacoeconomics 25, 899-902 (2007).

29 Schalekamp T, Oosterhof M, van Meegen E et al:: Effects of cytochrome P450 2C9 polymorphisms on phenprocoumon anticoagulation status. Clin. Pharmacol. Ther. 76, 409-417 (2004).

30 Schalekamp T, van Geest-Daalderop JHH, de Vries-Goldschmeding H, Conemans J, Bernsen MJ, de Boer A: Acenocoumarol stabilization is delayed in CYP2C93 carriers. Clin. Pharmacol. Ther. 75, 394-402 (2004).

31 Gale N, French DJ, Howard RL, McDowell DG, Debenham PG, Brown T: Rapid typing of STRs in the human genome by HyBeacon melting. Org. Biomol. Chem. 6, 4553-4559 (2008).

32 French DJ, Archard CL, Andersen MT, McDowell DG: Ultra-rapid DNA analysis using HyBeacon probes and direct PCR amplification from saliva. Mol. Cell. Probes 16, 319-326 (2002).

33 French DJ, Archard CL, Brown T, McDowell DG: HyBeacon probes: a new tool for DNA sequence detection and allele discrimination. Mol. Cell. Probes 15, 363-374 (2001).

34 Wadelius M, Pirmohamed M:

Pharmacogenetics of warfarin: current status and future challenges. Pharmacogenomics J 7 , 99-111 (2007).

35 Bush K, Kivlahan DR, McDonell MB, Fihn SD, Bradley KA: The AUDIT alcohol consumption questions (AUDIT-C): an effective brief screening test for problem drinking. Ambulatory Care Quality Improvement Project (ACQUIP). Alcohol Use Disorders Identification Test. Arch. Intern. Med. 158, 1789-1795 (1998).

36 Centre for Health Economics: EuroQol - a new facility for the measurement of health-related quality of life. The EuroQol Group. Health Policy 16, 199-208 (1990).

37 Schulman S, Kearon C: Definition of major bleeding in clinical investigations of antihemostatic medicinal products in non-surgical patients. J. Thromb. Haemost. 3 , 692-694 (2005).

38 Naranjo CA, Busto U, Sellers EM et al.: A method for estimating the probability of adverse drug reactions. Clin. Pharmacol. Ther. 30, 239-245 (1981). 
- Provides an objective model for estimation of the probability of adverse drug reactions.

39 Rosendaal FR, Cannegieter SC, van der Meer FJM, Briët E: A method to determine the optimal intensity of oral anticoagulant therapy. Thromb. Haemost. 69, 236-239 (1993).

40 Hillman MA, Wilke RA, Yale SH et al. A prospective, randomized pilot trial of model-based warfarin dose initiation using CYP2C9 genotype and clinical data. Clin. Med. Res. 3, 137-145 (2005).

II Reports the first randomized trial of a genotype-guided warfarin dosing algorithm. Although some effects were observed, power was insufficient to compare outcomes.

41 Anderson JL, Horne BD, Stevens SM et al.: Randomized trial of genotype-guided versus standard warfarin dosing in patients initiating oral anticoagulation. Circulation 116, 2563-2570 (2007).

- - Reports the first clinical trial which included both CYP2C9 and VKORC1 polymorphisms in the dosing algorithm, which shows an improvement during warfarin therapy initiation.

42 Caraco Y, Blotnick S, Muszkat M: CYP2C9 genotype-guided warfarin prescribing enhances the efficacy and safety of anticoagulation: a prospective randomized controlled study. Clin. Pharmacol. Ther. 83, 460-470 (2008).

43 Kangelaris KN, Bent S, Nussbaum RL, Garcia DA, Tice JA: Genetic testing before anticoagulation? A systematic review of pharmacogenetic dosing of warfarin. J. Gen. Intern. Med. 24, 656-664 (2009).

44 Palareti G, Leali N, Coccheri S et al.: Bleeding complications of oral anticoagulant treatment: an inception-cohort, prospective collaborative study (ISCOAT). Italian Study on Complications of Oral Anticoagulant Therapy. Lancet 348, 423-428 (1996).
45 Poli D, Antonucci E, Lombardi A et al.: Low rate of bleeding and thrombotic complications of oral anticoagulant therapy independent of age in the real-practice of an anticoagulation clinic. Blood Coagul. Fibrinolysis 14, 269-275 (2003).

46 Hylek EM, Chang YC, Skates SJ,

Hughes RA, Singer DE: Prospective study of the outcomes of ambulatory patients with excessive warfarin anticoagulation. Arch. Intern. Med. 160, 1612-1617 (2000).

47 Odén A, Fahlén M: Oral anticoagulation and risk of death: a medical record linkage study. BMJ 325, 1073-1075 (2002).

48 Ansell J, Hirsh J, Hylek E, Jacobson A, Crowther M, Palareti G: Pharmacology and management of the vitamin $\mathrm{K}$ antagonists: American College of Chest Physicians Evidence-Based Clinical Practice Guidelines (8th Edition). Chest 133, 160S-198S (2008).

\section{Websites}

101 Federation of Dutch Anticoagulant Clinics, Summary of medical annual report (2007) www.fnt.nl/pdf/FNT\%20Medisch\%20 Jaarverslag\%202007\%20FC.pdf (in Dutch)

102 ICH Harmonised Tripartite Guideline Guideline for Good Clinical Practice E6(R1) www.emea.europa.eu/pdfs/human/ ich/013595en.pdf

103 Curtis L, Netten A: Units costs of health and social care. (2005) http://www.pssru.ac.uk

104 Transcript of the FDA press conference on Warfarin held on 16 August 2007. www.fda.gov/downloads/NewsEvents/ Newsroom/MediaTranscripts/ucm123583.pdf

105 Website of the clarification of optimal anticoagulation through genetics research network http://coagstudy.org/

\section{Affiliations}

- Rianne MF van Schie

Utrecht University, Faculty of Science, Utrecht, The Netherlands

- Mia Wadelius

Uppsala University, Uppsala, Sweden

- Farhad Kamali

Newcastle University, Newcastle upon Tyne, UK

- Ann K Daly

Newcastle University, Newcastle upon Tyne, UK

- Vangelis G Manolopoulos Democritus University of Thrace, Alexandroupolis, Greece

- Anthonius de Boer Utrecht University, Faculty of Science, Utrecht, The Netherlands

- Rita Barallon LGC Limited, Middlesex, UK

- Talitha I Verhoef Utrecht University, Faculty of Science, Utrecht, The Netherlands

- Julia Kirchheiner Humboldt University of Berlin, Berlin, Germany

- Elisabeth Haschke-Becher Elisabethinen Hospital Linz, Linz, Austria

- Montserrat Briz Hospital Sierrallana-IFIMAV, Cantabria, Spain

- Frits R Rosendaal Leiden University Medical Center, Leiden, The Netherlands

- William K Redekop Erasmus University Medical Center, Rotterdam, The Netherlands

- Munir Pirmohamed University of Liverpool, Liverpool, UK

- Anke-Hilse Maitland-van der Zee Utrecht University, Faculty of Science, Division of Pharmacoepidemiology and Pharmacotherapy, Utrecht, The Netherlands 